\title{
Redistributed Records Collections Personality Matching using Encrypted Cryptosystem
}

\author{
R. Velvizhi , C. Rajabhushanam, S.R. Sri Vidhya
}

\begin{abstract}
Sensitive information is gradually distributed in the cloud in this project's cloud computing and processing services to reduce costs, which raises concerns regarding data privacy. Encryption was a positive way to keep outsourced sensitive data secure, but it makes efficient use of data a very difficult process.

In this paper, we focus on the issue of private matching in ide ntity-based cryptosystem over outsourced encrypted data sets that can simplify the management of certificates. To solve this proble $m$, we are proposing a private matching scheme based on identity
\end{abstract}

Keyword-Cloud computing, Data mining, Data sharing, Cloud service provider.

\section{INTRODUCTION}

Portable wellbeing (mHealth) advances, including remote observing, wearable gadgets, and installed sensors, have developed quickly in the previous years and demonstrated incredible potential to improve the quality and effectiveness of medicinal services. In mHealth, long haul and consistent wellbeing checking is empowered by cell phones that remotely interface biomedical sensors. The biomedical sensor check be made to be light, sturdy, and agreeable easily and can detect an enormous assortment of biomedical sign or physical exercises, for example, electrocardiogram, glucose focus, breathing rate, beat rate, circulatory strain, fringe oxygen immersion, and body movement A case of such biomedical sensors is the "biostamp" structured by an organization called MC10,which is quarter-size, waterproof, and breathable, and costs only many pennies under cluster creation The detected information can be transmitted to a remote mHealth server, which conducts analysison the biomedical information and returns convenient advices to the detected subject. Wellbeing observing through biomedical sensors empowers convenient mediation and better administration of individual wellbeing status, in this manner essentially improving medicinal services quality.

Detecting incorporate the two patients and solid individuals. The information of sound individuals are not accessible in conventional social insurance since therapeutic information are possibly gathered when patients visit centers.

Revised Manuscript Received on October 22, 2019.

* Correspondence Author

R. Velvizhi*, Department of Computer Science and Engineering, Bharath Institute of Higher education and research, Chennai , India

C. Rajabhushanam, Department of Computer Science and Engineering, Bharath Institute of Higher education and research, Chennai, India

S.R. Sri Vidhya, Department of Computer Science and Engineering, Bharath Institute of Higher education and research, Chennai , India

Notwithstanding, biomedical information from sound individuals can be utilized as positive examples for preparing prescient models and will include significant in-sights of malady aversion and expectation. Second, since biomedical sensors can screen the human body day and night over along time length, the information gathered by biomedical sensors have a lot bigger volume than conventional restorative information.

At this scale empower fine-grained determination and treatment, for example, customized drug, and may to a great extent improve human services quality and proficiency. Because of the tremendous capability of biomedical detecting information in medicinal services, analysts from the Institute of System Biology have started a task called 100K Wellness Project, which means to strongly screen 100; 000 solid people and watch their physiology for a long time . It is imagined that examination on huge scale biomedical detecting information will uncover the soonest harbingers of executioner infections, for example, malignant growth and coronary illness. In this paper, we center around calculated relapse, an exemplary AI strategy which is proper for anticipating dichotomous results and in this way broadly utilized for settling on choices in therapeutic analysis and visualization.

\section{Literature Survey}

\section{A.Hierarchical Attribute-Based Encryption for Fine-Grained Access Control in Cloud Storage Services}

The creators recommend that freely auditable cloud information stockpiling we propose a plan to help ventures to effectively share secret information on cloud servers. We accomplish this objective by first joining the progressive character based encryption (HIBE) framework and the figure content arrangement characteristic based encryption (CP-ABE) framework, and afterward making an exhibition expressivity tradeoff, at long last applying intermediary re-encryption and lethargic re-encryption to our plan.

Disadvantages: The existing CP-ABE schemes usually demand users to heavily depend on AAs and maintain a large amount of secret keys storage, which lacks flexibility and scalability 


\section{B. Cipher text-Policy Attribute-Based Encryption}

The creators propose a this paper we present a framework for acknowledging complex access control on scrambled information that we call Ciphertext-Policy Attribute-Based Encryption. By utilizing our systems scrambled information can be kept private regardless of whether the capacity server is untrusted; also, our strategies are secure against agreement assaults.

Disadvantages: Most existing public key encryption method allow a party to encrypt data to a particular user, but are unable to efficiently handle more expressive types of encrypted access control

\section{OBJECTIVE}

In this project primary hospital maintain the sensitive illness symptoms and medications of the patients in their database and move the corresponding data into cloud . If an emergency situation, other hospital needs to know about the specific patient details, they can easily get data after getting access key from the primary hospital. In this scenario the cloud server acts as the central hierarchy who contains the data which is uploaded by primary hospital. In this case secondary hospital uploads any data which is related to the corresponding patient, the data gets stored at the same cloud server.

\section{EXISTING METHOD}

The current Web-Based Information System for In numerous applications, private coordinating isn't constantly exact or full because of info mistakes, oversights or conflicting spelling of names. In these cases, it is valuable to have a private coordinating calculation that reports a match regardless of whether two informational collections are not coordinated precisely. Next, we present two applications f our fundamental personality based cryptosystem plot, in particular character based fluffy private coordinating and personality multi-watchword fluffy inquiry.

1. In existing system to matching request no matching response.

2. Not implemented hierarchical method to data sets

3. In this method not use any two sectors to matching accurately.

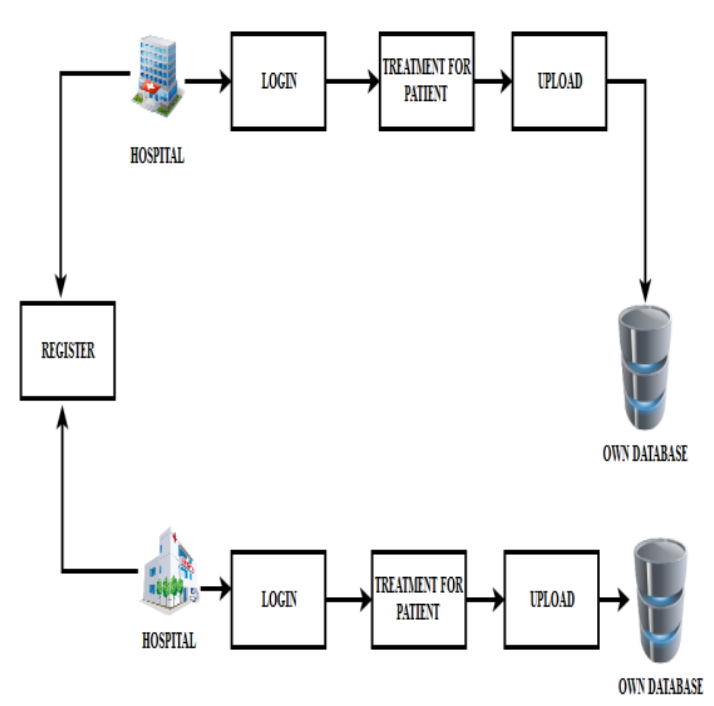

\section{V.PROPOSED METHOD}

The development of this new system contains the following activities, which try to recover the problems from the previous system.

1. The website will display information about all the hospitals and the patients including their treatment details.

2. Provides facility for instance search for patients provides registration for the users to ensure security.

3. Requests for various patients details are accepted and granted

4. The administrator can ensure the system to be more informative adding a hospitals

1. In this proposed system to use globalized data sets we can access any time any ware

2. In this system to implement an Identity-Based Private Matching scheme

3. To give matching request matching response. To easily access data.

4. Easily search option implemented to access data.

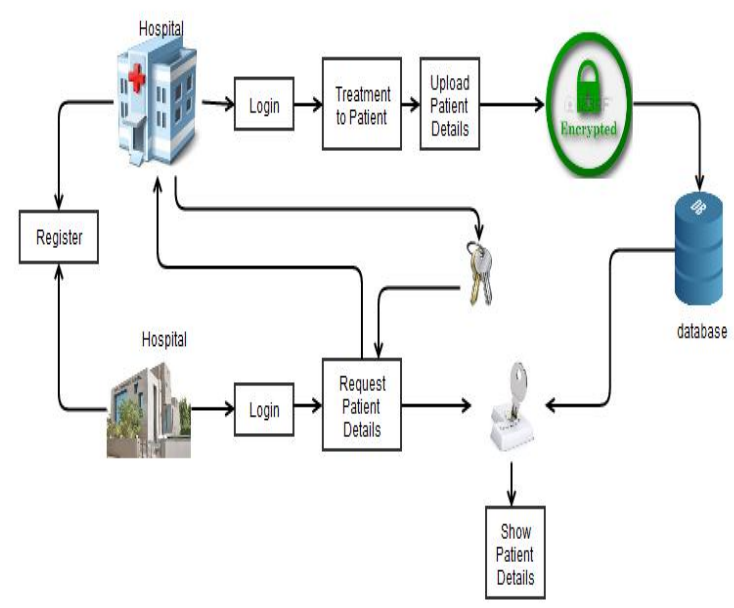




\section{RESULTS}

The system after careful analysis has been identified to be presented with the following modules:

1. Search module

2. Authentication

3. Key distribution

4. Files sharing

5. Matching request, Matching result

6. Authorization token

7. Negotiation

\section{Search module:}

The process of finding a detail from an overview database plays a major role. To retrieve the data from that is a different part but searching the data in a database need to be get focused in term of finding out the available data in it. This search module helps to find the required data from the database based on the keyword specified.

\section{Authentication:}

To retrieve data from the database the user who were requesting the data should be verified in term of authentication process to avoid data leakage to invalid user. The user request get processed only when the user is authorized to the particular network

\section{Key Distribution:}

The key distribution process done only after the user is consider to be valid in term of authentication. The key is provided by the admin to make the user to retrieve the data from the database. The key access is provided to make the user as authorized one for data access.

\section{Files sharing:}

The file get shared to the user in term of data sharing in which the data get shared to number of valid user for data usage. The file will be shared to the user who were having the valid key of that particular data. The file get shared to the user once the file access key will be changed to avoid repetitive data retrieval by the same user in the database.

\section{Matching request, Matching result :}

Matching request will works to check out the request matching to check whether the request has been already arrived from any other user. The data retrieval process will be easy if the request is already exists by any other user. The data retrieving process is easy if the data retrieving path will be already exists one. Matching result is used to check whether the data retrieved from the database exactly matches the request that is process by the concern user who were requested for the data.

\section{Authorization token:}

Authorized token will be issued to the users who were retrieved the data from the database. It is used to reduce the user authentication process once again user request the database for different data to retrieved from the database. Making use of the authorized token the data requesting process also be a easier one so that the data retrieval process will be a quick term work.

\section{Negotiation:}

Negotiation process used to avoid the user request which processed as an unwanted one for data retrieval from the database. The data will be stored as a secured one to protect the data from the unwanted user who were requesting for data that are not related or it is not in their authorization. The negotiation process used to neglect the user or to terminate the user from that particular network.

\section{CONCLUSION}

In this project we mainly focused on secure data sharing between two users. When sharing the data ,the data owner will select the users with whom he wants to share and then while sharing the data, data owner will encrypt the data and he will share the data and the key to the cloud. Then the data user send request for key to cloud. By using that key data user can get the original data.

\section{REFERENCES}

1. Anderson, H., Suzuki, Q., and Zhao, B. Deploying DHTs and access points. Journal of Wireless Information 84 (Sept. 2001), 88-107.

2. Gupta, K. Controlling Moore's Law and the Internet. Journal of Knowledge-Based, Metamorphic Symmetries 38 (Nov. 2005), 1-16.

3. Ito, I. Emulating Scheme using multimodal archetypes. In Proceedings of NSDI (Dec. 2003).

4. Kubiatowicz, J., and Kubiatowicz, J. Synthesizing systems and the Internet. In Proceedings of PODC (July 2003).

5. Qian, P. J. Improving reinforcement learning and Internet QoS In Proceedings of SIGMETRICS (Mar. 1994).

6. Ritchie, D., and White, N. Contrasting checksums and SCSI disks with Maulstick. In Proceedings of JAIR (Sept. 2001).

7. Thomas, U. Autonomous, linear-time epistemologies. In Proceedings of the Workshop on Semantic Archetypes (July 2003).

8. Thompson, K. Visualizing superblocks and write-ahead logging using Paper. In Proceedings of the USENIX Security Conference (Dec. 2004).

9. Williams, N. The effect of certifiable technology on algorithms In Proceedings of the Workshop on Ubiquitous, Constant-Time Modalities (Apr. 1999).

10. Wilson, Z., Sun, E., Culler, D., Robinson, R., Raman, L., Einstein, A., Sutherland, I., and Takahashi, Q. Z. Heterogeneous, metamorphic technology for local-area networks. In Proceedings of the Conference on Secure, Semantic Configurations (Aug. 2004).

11. Zhou, O. C., and Wilkinson, J. Highly-available, flexible theory for B-Trees. In Proceedings of the Workshop on Replicated Epistemologies (Jan. 2004).

\section{AUTHORS PROFILE}

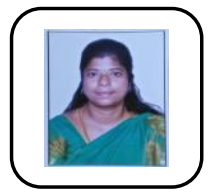

R. Velvizhi, Assistant Professor, Department of Computer Science \& Engineering, Bharath Institute of Higher Education and Research, Chennai, India

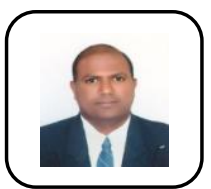

C. Rajabhushanam, Associate Professor, Department of Computer Science \& Engineering, Bharath Institute of Higher Education and Research, Chennai, India

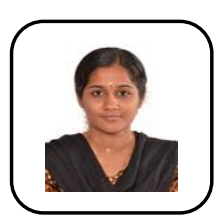

S.R. Sri Vidhya, Assistant Professor, Department of Computer Science \& Engineering, Bharath Institute of Higher Education and Research, Chennai, India 